\title{
Analysis of Force Distribution of Four Rows of Bolts in Aircraft Fitting Joint
}

\author{
Yifang Sun $\mathbb{D}^{1}{ }^{1}$ O. G. Grebenikov, ${ }^{1}$ and Chenghu $\mathrm{Li}(\mathbb{D})^{2,3}$ \\ ${ }^{1}$ National Aerospace University "Kharkiv Aviation Institute", 61070 Kharkiv, Ukraine \\ ${ }^{2}$ School of Aerospace Engineering, Huazhong University of Science and Technology, 430074 Wuhan, China \\ ${ }^{3}$ School of Aircraft Engineering, Nanchang Hangkong University, Nanchang 330063, China
}

Correspondence should be addressed to Chenghu Li; lichenghu111@126.com

Received 11 March 2021; Revised 12 June 2021; Accepted 29 June 2021; Published 14 July 2021

Academic Editor: Rosario Pecora

Copyright (c) 2021 Yifang Sun et al. This is an open access article distributed under the Creative Commons Attribution License, which permits unrestricted use, distribution, and reproduction in any medium, provided the original work is properly cited.

With the help of 3D modeling software and finite element analysis software, the force distribution of four rows of bolts in the aircraft fitting joint is obtained in this paper. In 3D modeling software, the solid model is segmented according to the area and height of the center of gravity of the cross-section. In finite element analysis software, the beam elements are used to replace the segmented model to obtain the internal force applied at the end of the segmented model and the stress-strain state of each segment is calculated according to the internal force applied at the end of each segment. Finally, the force distribution of the four rows of bolts is obtained according to the average force difference on the center section between the bolt rows. In order to ensure the validity of this step-by-step calculation result, it was compared with the entire calculation result. It can be concluded that the force distribution of the two calculations is consistent. Under the working conditions of the fitting joint (130 MPa), the force distribution coefficients of the four rows of bolts are $0.182,0.215,0.197$, and 0.12 . Therefore, it is feasible to use the stepby-step method to calculate the force distribution of the four rows of bolts in the fitting joint. Compared with the entire calculation, the step-by-step calculation has the advantages of less calculation amount and faster calculation speed.

\section{Introduction}

Fitting joints are widely used in aircraft structures and are responsible for the interconnection of important components, such as the connection between the center wing section and outer wing section and the connection between the horizontal and vertical tail and the fuselage. Once these fitting joints are damaged, the aircraft will not be able to fly in the sky and the consequences will be very grievous. Therefore, the design and analysis of fitting joints are one of the most important issues in aircraft design. The fitting joints are usually connected by bolts. For multirow bolted fitting joints, the force distribution between each row of bolts directly affects the performance of the fitting joint. A simple method to calculate the force distribution between each row of bolts in the fitting joint should be developed to improve the design efficiency of the fitting joint and guide the selection and installation of bolts. However, the force transmission in the fitting joint is very complicated, which is due to the complicated structure of the fitting joint, so it is difficult to analyze the performance of the fitting joint. With the development of numerical methods and the application of finite element software, it becomes possible to analyze the entire fitting joint and each component. This article focuses on the calculation of the force distribution between bolt rows based on the analysis of the stress-strain state of fitting joint.

Relevant scholars have done a lot of research to analyze the performance of fitting joints. Vasilevsky et al. [1-3] analyzed the overall stress-strain state of the fitting joint of the center wing section by a segmented and simplified method. Then, the stress-strain state of each component was obtained according to the overall stress-strain state of the fitting joint. Wang et al. $[4,5]$ designed a special joint to withstand radial loads and checked the strength of the designed wing joint through entire finite element analysis. The results showed that the strength of the designed joint can fully meet the test requirements. Qin et al. [6] carried out failure analysis on a certain type of aircraft wing-fuselage joint fatigue specimens 
through methods such as fracture macro/micro observation, metallographic inspection, finite element numerical analysis, and test implementation inspection. The results show that it is the load frequency, the asynchrony of the loads, and the insufficient constraints of the web plate that cause greater stress at the connecting bolt of the web, leading to the initiation of fatigue crack and the short fatigue life of the experimental samples. It provides a good reference for the redesign of the fitting joint. Zhao and Wei [7] used the elastoplastic contact analysis module of MARC, a large-scale finite element software, to perform nonlinear coupled analysis on the wing-fuselage joints with continuously refining mesh. Finally, the elastic-plastic contact finite element model of the butt joint is created, and stress distribution and the yield condition of the joint are obtained based on the nonlinear finite element method. Wang et al. [8] combined the standard finite element program with the improved mean value method to analyze the stiffness reliability of the wingfuselage attachment joint of a certain type of aircraft. The results indicate that at applied load and allowed strain, the joint structure has higher reliability when coefficient of variation in applied load is 0.15 .

In order to decrease computing time and resources, Liu and Chen [9] proposed a two-dimensional to threedimensional global-local finite element numerical method, in which both the local zone selection strategy and boundary constraints applying method are discussed in detail. And both the contact stress and the stress components around the fastener hole are analyzed by using the proposed globallocal method. Egan et al. [10] used the nonlinear finite element code Abaqus to model single-lap carbon-epoxy joints with countersunk fasteners and performed a highly detailed analysis of the stress distribution at the countersink hole boundary. Ascione [11] presented the first results of a numerical and experimental investigation on the shear force distribution in a bolted joint made entirely from FRP materials and proposed an experimental equipment for investigating the strains and stress distributions around the holes of the connection as well as the bearing stresses at the interface between plate and steel bolt. Xiang et al. [12] developed an improved spring method considering the hole clearance and friction effects for efficient modeling of the composite bolted joint structures. The results show that the proposed method is time efficient for the load distribution prediction of the bolted joint composite structures. Konkong and Phuvoravan [13] studied the influence of geometric variables on the load distribution of cold-formed steel bolt connections. The results of the parametric study showed that the $t_{2} / t_{1}$ ratio controlled the efficiency of the bolt load distribution more than the other parameters studied.

It can be seen from the above researches that obtaining the stress-strain state of the fitting joint is an important way to analyze the performance of the fitting joint. In this paper, based on Vasilevsky et al.'s and Egan et al.'s research $[1-3,10]$, the stress-strain state of the fitting joint of the outer wing section will be calculated. According to the stress state of the fitting joint of the outer wing section, the force distribution between the bolt rows is analyzed in detail.
In this article, the fitting joint between the center wing section and the outer wing section of the aircraft AN-148 [14] will be taken as the analysis object. Figure 1 shows the center wing section and outer wing section of the aircraft AN-148. It can be seen that the center wing section and outer wing section are connected by the fitting joint. The connection between the center wing section and the outer wing section is the fitting joint. The structure of the fitting joint is very complicated, so the model of the fitting joint of the outer wing section should be simplified for analysis.

Due to the numerous, complex structures of components and variable types of such fitting joint, experiment cost is high and the cycle is long and so cannot meet the manufacturing requirements. An effective and reasonable method is to analyze the stress-strain state of the fitting joint step by step and then analyze the force distribution between the bolt rows according to the stress state. The detailed execution steps for calculating the force distribution between bolt rows are shown in Figure 2.

\section{Segment and Simplify 3D Model of Fitting Joint}

The connection between the center wing section and the outer wing section can be seen as a combination of many similar structural units. For these similar structural units, the same method can be used to calculate their stress-strain state and the force distribution between bolt rows. In this paper, the single connecting unit in the middle below between the center wing section and the outer wing section is taken as the research object for analysis. The fitting joint structure of a single connection unit is shown in Figure 3. This structure includes fitting of center wing section (FCWS), intermediate panel (IP), fitting of outer wing section (FOWS), stringer of outer wing section (SOWS), lower panel of outer wing section (LPOWS), hexagon head bolts (M18), countersunk head bolts (M8 and M6), and countersunk head rivets (M6), as shown in Figures 3. The equivalent stress of the gross load at the end of the stringer of outer wing section is $\sigma_{\text {eq }}=130 \mathrm{MPa}$. In the total section of the regular area, the load $F$ on the end section can be obtained by the following formula:

$$
F=\sigma_{\mathrm{eq}} \cdot S_{\mathrm{sec}}
$$

where $S_{\text {sec }}$ is the area of the cross-section.

The fitting of center wing section, intermediate panel, fitting of outer wing section, lower panel of outer wing section, and stringer of outer wing section are all made of D16T aluminum alloy. The main parameters of the D16T aluminum alloy are shown in Table 1. The hexagon head bolts (M18) and countersunk head bolts (M8 and M6) are all made of VT6 titanium alloy. The main parameters of the VT6 titanium alloy are shown in Table 2.

In the three-dimensional model, the cross-sectional shapes at different positions of the model can be obtained by transverse cutting. According to the different crosssectional shapes, 15 different cross-sections are determined, 


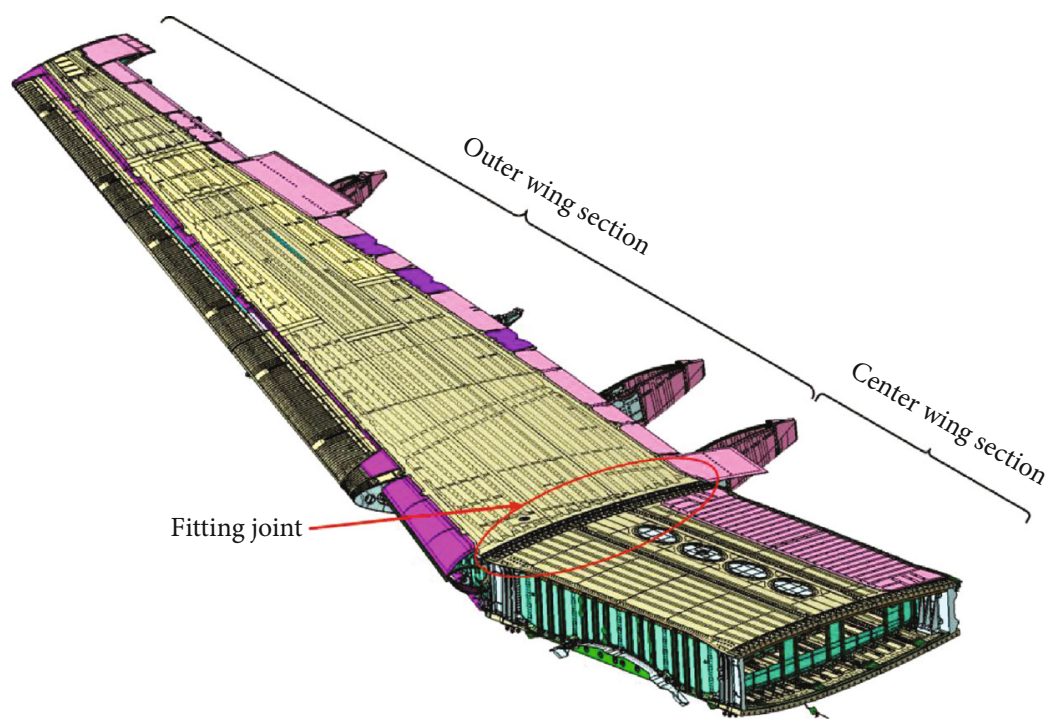

FIGURE 1: The fitting joint between the center wing section and the outer wing section of the aircraft AN-148.

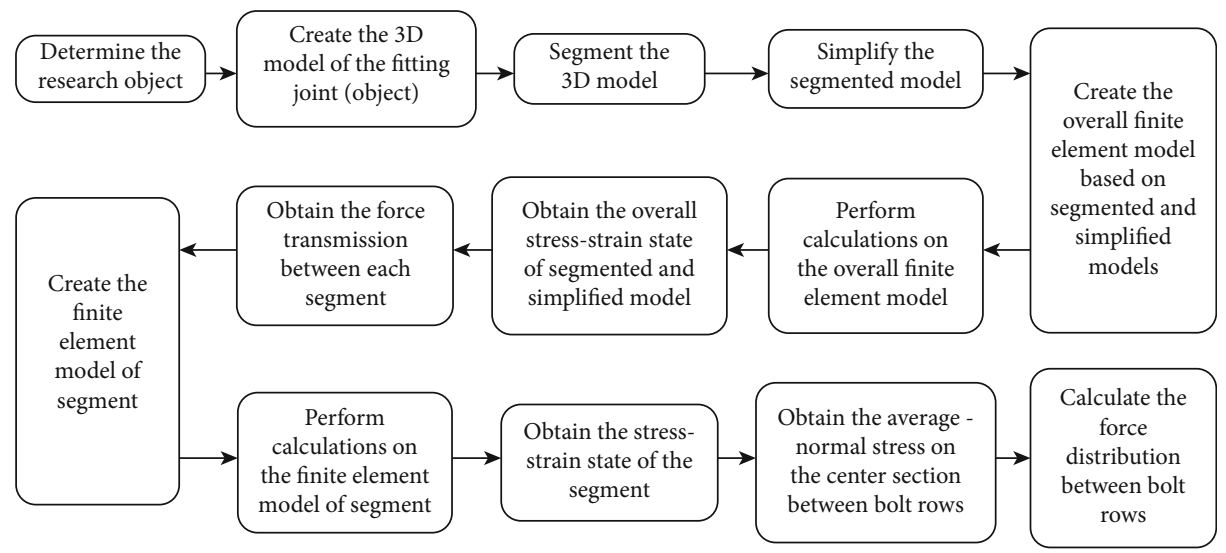

FIGURE 2: The execution steps for calculating the force distribution between bolt rows.

which divide the fitting joint into 15 segments as shown in Figure 4 . These 15 segments reflect the characteristics of the cross-sectional geometry (changes in cross-sectional shape) and the structure and force characteristics of the design model (moment of inertia and position of the center of gravity). In order to simplify each segmented model, each segmented model is regarded as a whole and beam elements are used to replace it. In Figure 4, (a) shows a schematic diagram of the longitudinal section of the fitting joint, (b) shows a schematic diagram of the segmented model of the fitting joint, (c) shows a schematic diagram of the simplified model of the fitting joint, (d) shows a schematic diagram of the area of different cross-sections, and (e) shows a schematic diagram of the height of the center of gravity of different cross-sections.

\section{Finite Element Analysis of the Segmented and Simplified Model}

In order to obtain the transmitted force at the two ends of each segment, the segmented and simplified models of the fit- ting joint are analyzed using the finite element analysis software (Workbench). In the process of establishing the finite element model, each segmented model is regarded as a whole and replaced with a finite element BEAM188. The general finite element model of the fitting joint is created as shown in Figure 5. The point $L_{0}$ is a fixed constraint, the points $L_{555}, L_{966}, L_{1700}$, and $L_{2200}$ are simply supported constraints (impose $y$-axis direction constraints), and the point $L_{2200}$ bears a horizontal load $F=135200 \mathrm{~N}$. In the calculation, a cross-sectional shape is assigned to each segment, and the assigned cross-sectional shape is the same as the crosssectional shape obtained by transverse cutting at different positions of the three-dimensional model. When the gross load is $130 \mathrm{MPa}$, the calculation results of shear force, bending moment, displacement, and axial force are shown in Figures 6-9.

BEAM188 is suitable for analyzing slender to moderately stubby/thick beam structures. The element is based on the Timoshenko beam theory which includes sheardeformation effects. The element provides options for unrestrained warping and restrained warping of cross-sections. 


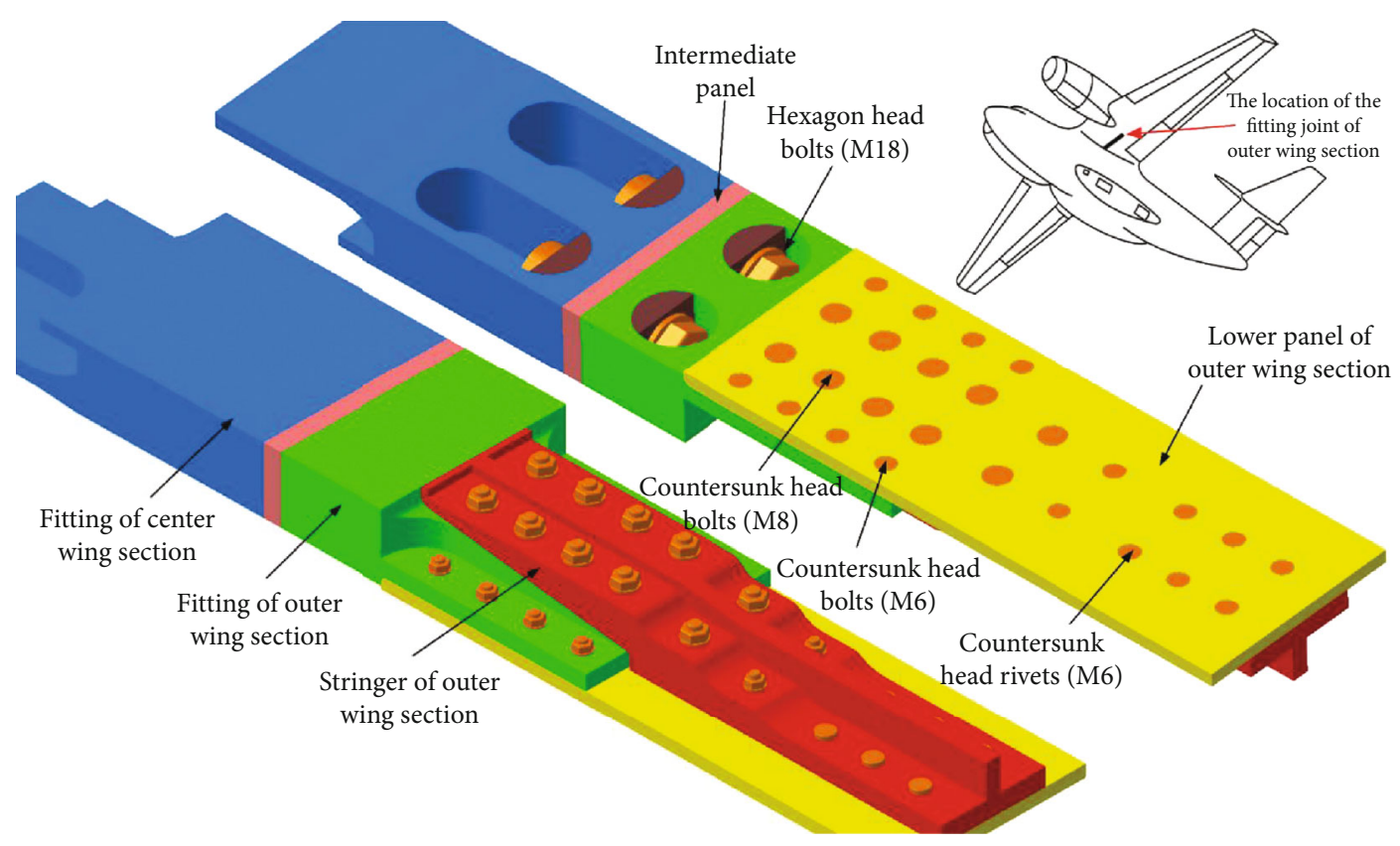

Figure 3: The 3D model of the fitting joint.

TABLE 1: The main parameters of D16T aluminum alloy.

\begin{tabular}{lcccc}
\hline Density & Young's modulus & Tensile ultimate strength & Tensile yield strength & Poisson's ratio \\
\hline $2780\left(\mathrm{~kg} / \mathrm{m}^{3}\right)$ & $72(\mathrm{GPa})$ & $440(\mathrm{MPa})$ & $300(\mathrm{MPa})$ & 0.33 \\
\hline
\end{tabular}

TABLE 2: The main parameters of VT6 titanium alloy.

\begin{tabular}{lcccc}
\hline Density & Young's modulus & Tensile ultimate strength & Tensile yield strength & Poisson's ratio \\
\hline $4450\left(\mathrm{~kg} / \mathrm{m}^{3}\right)$ & $115(\mathrm{GPa})$ & $1100(\mathrm{MPa})$ & $835(\mathrm{MPa})$ & 0.35 \\
\hline
\end{tabular}

The element is a linear, quadratic, or cubic two-node beam element in 3D. BEAM188 has six or seven degrees of freedom at each node. These include translations in the $x, y$, and $z$ directions and rotations about the $x, y$, and $z$ directions. A seventh degree of freedom (warping magnitude) is optional. This element is well-suited for linear, large rotation, and/or large strain nonlinear applications.

Therefore, the shear force at each position of the segmented and simplified model can be obtained from Figure 6 and the end of the 10th segment has a shear force of $-2767.6 \mathrm{~N}$. The bending moment at each position of the segmented and simplified model can be obtained from Figure 7 and the end of the 10th segment bears a bending moment of $129775 \mathrm{~N} \cdot \mathrm{mm}$. The displacement of each position of the segmented and simplified model can be obtained from Figure 8 and the end of the 10th segment has a displacement of $1.0257 \mathrm{~mm}$. The axial force at each position of the segmented and simplified model can be obtained from Figure 9, and the end of the 10th segment bears an axial force of $135190 \mathrm{~N}$.

\section{Finite Element Analysis of the Single Segment}

The four-row bolt connection structure is only in the outer section of the wing, so only the 10th segment model needs to be selected for analysis. The 10th segment model is composed of fitting of outer wing section, stringer of outer wing section, lower panel of outer wing section, and countersunk head bolts (M8 and M6) as shown in the 3D model in Figure 10. According to the results of finite element analysis of the segmented and simplified model, the boundary conditions of the fitting joint of outer wing section can be obtained and then the detailed stress-strain state of the fitting joint of outer wing section can be calculated using finite element software (Workbench). The three-dimensional model is imported into Workbench through the intermediate format STP, and the finite element model is obtained as shown in Figure 11.

The finite element model of the local fitting joints should be constrained by the following boundary conditions according to the actual working conditions and the overall stressstrain state results (Figures 6-9) of the fitting joint.

(1) The bolts are also subjected to axial pretightening force, the pretightening force of the M8 bolt is $12400 \mathrm{~N}$, and the pretightening force of the M6 bolt is $6750 \mathrm{~N}$

(2) The contacts between all the bolts and fitting of center wing section, intermediate panel, fitting of outer 


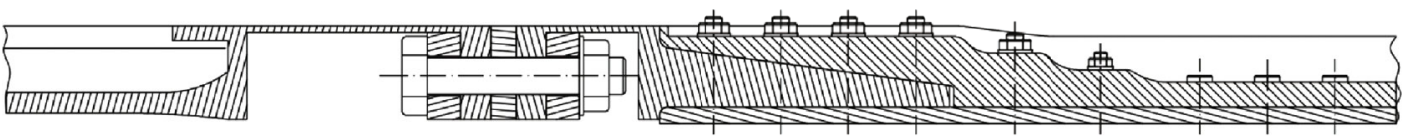

(a)

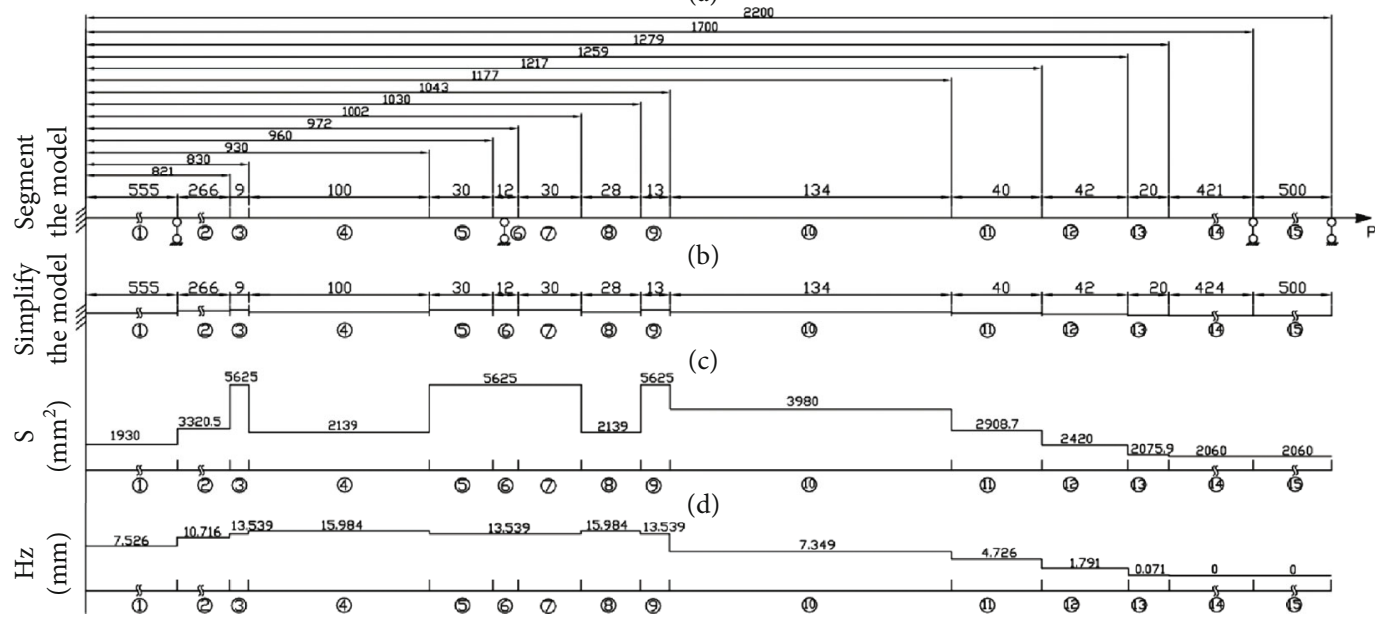

FIGURE 4: The segmentation and simplification of the model of the fitting joint. (a) The longitudinal section of the fitting joint; (b) the position of different cross-sections; (c) the segmented models of the fitting joint; (d) The area of different cross-sections; (e) the height of the center of gravity of different cross-sections.

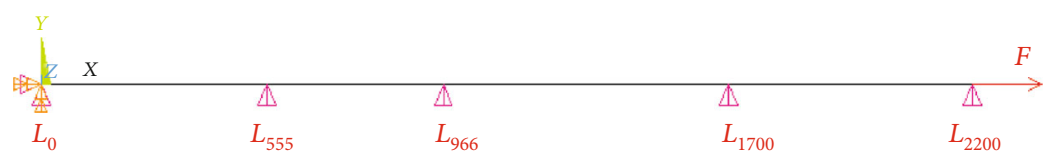

FIgURE 5: The finite element model in Workbench.

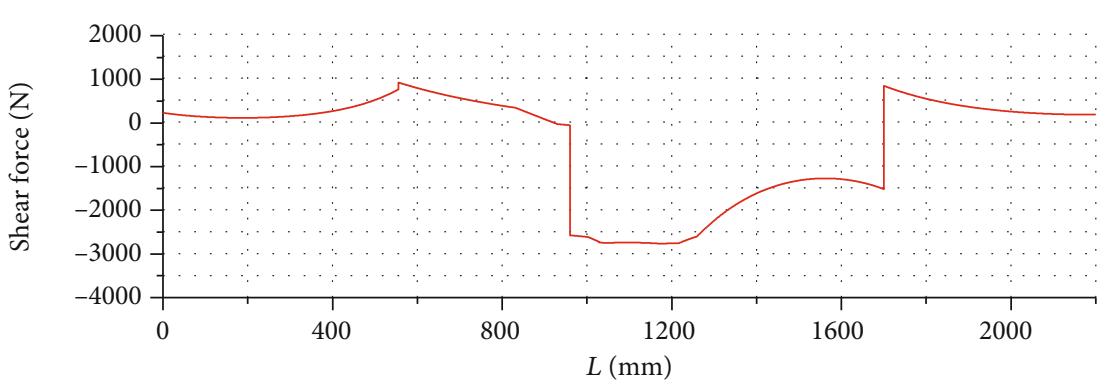

Figure 6: Shear force diagram when the gross load is $130 \mathrm{MPa}$.

wing section, stringer of outer wing section, and lower panel of outer wing section are frictionless. The contacts between all the nuts and fitting of center wing section, fitting of outer wing section, and stringer of outer wing section are frictional with friction coefficient of 0.3 (determined according to the technological requirements of aircraft AN148)

(3) The contacts between the fitting of center wing section, fitting of outer wing section, stringer of outer wing section, and lower panel of outer wing section are frictional contact, and the friction coefficient is 0.3

(4) The constraints of the cutting surfaces on both sides of the model of overall fitting joints are frictionless. The constraint of the rear-end cutting surface of the model of the overall fitting joint is fixed
(5) The end of the 10th segment has a shear force of $-2767.6 \mathrm{~N}$, a bending moment of $259550 \mathrm{~N} \cdot \mathrm{mm}$, and an axial force of $135190 \mathrm{~N}$. The shear force here is caused by the different positions of the center of gravity of the segments of the fitting joint, and partial bending occurs due to eccentricity during load transmission

The stress state of the outer wing segment in the 10th segment model is shown in Figure 12. It also shows the average normal stress of the center section between each bolt row.

As shown in Figure 12, the a-a, b-b, c-c, d-d, and e-e sections are obtained by cutting the fitting of outer wing section in the 10th segment. The average stress on the a-a section is 44.647 $\mathrm{MPa}$, the average stress on the b-b section is 43.206 MPa, the average stress on the $\mathrm{c}-\mathrm{c}$ section is 


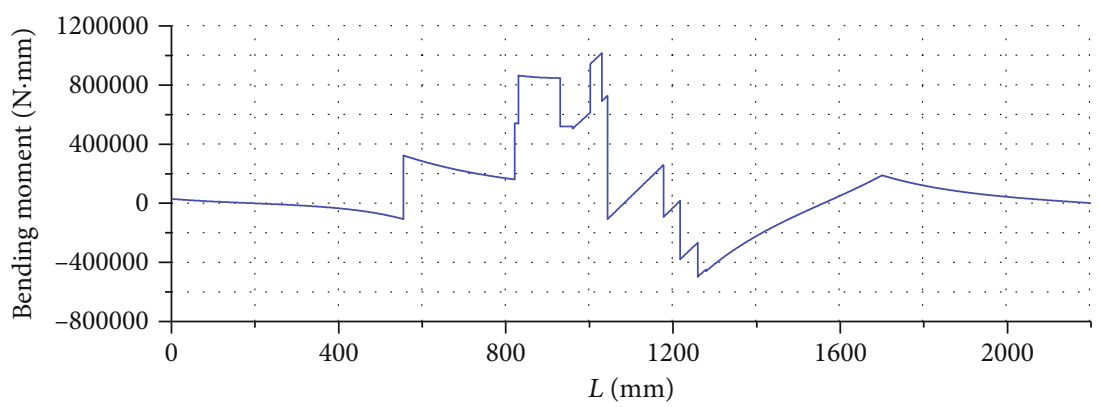

FIgURE 7: Bending moment diagram when the gross load is $130 \mathrm{MPa}$.

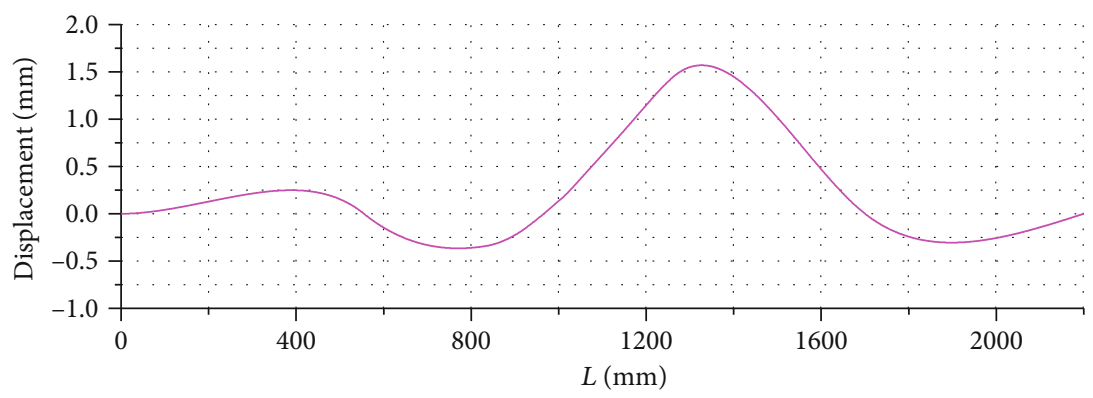

Figure 8: Displacement diagram when the gross load is $130 \mathrm{MPa}$.

34.117 MPa, the average stress on the $\mathrm{d}$ - $\mathrm{d}$ section is $25.0226 \mathrm{MPa}$, and the average stress on the e-e section is 19.556 MPa.

\section{Finite Element Analysis of the Entire Fitting Joint}

When calculating the stress-strain state of the fitting joint, the 3D model of the entire assembly joint is directly imported into Workbench for calculation without the need to simplify the model. Then, meshing and boundary condition settings are performed in Workbench and the finite element model of the fitting joint is obtained. The three-dimensional model of the fitting joint is shown in Figure 3. The finite element model of the fitting joint is shown in Figure 13. It also shows some finite element models of bolts and bolt holes.

The finite element model of the fitting joints should be constrained by the following boundary conditions according to the actual working conditions:

(1) The bolts are also subjected to axial pretightening force, the pretightening force of the M18 bolt is $66000 \mathrm{~N}$, the pretightening force of the M8 bolt is $12400 \mathrm{~N}$, and the pretightening force of the M6 bolt is $6750 \mathrm{~N}$

(2) The contacts between all the bolts and fitting of center wing section, intermediate panel, fitting of outer wing section, stringer of outer wing section, and lower panel of outer wing section are frictionless. The contacts between all the nuts and fitting of center wing section, fitting of outer wing section, and stringer of outer wing section are frictional with fric- tion coefficient of 0.3 (determined according to the technological requirements of aircraft AN148)

(3) The contacts between the fitting of center wing section, fitting of outer wing section, stringer of outer wing section, and lower panel of outer wing section are frictional with friction coefficient of 0.3

(4) The constraints of the cutting surfaces on both sides of the model of overall fitting joints are frictionless. The constraint of the rear-end cutting surface of the model of overall fitting joint is fixed

(5) The end of the stringer of outer wing section has a force of $135200 \mathrm{~N}$

The stress state of the entire fitting joint is shown in Figure 14. It also shows the average normal stress of the center section between each bolt row.

As shown in Figure 14, the a-a, b-b, c-c, d-d, and e-e sections are obtained by cutting the fitting of outer wing section in the 10th segment. The average stress on the a-a section is $45.562 \mathrm{MPa}$, the average stress on the $\mathrm{b}-\mathrm{b}$ section is $45.537 \mathrm{MPa}$, the average stress on the $\mathrm{c}-\mathrm{c}$ section is 36.419 MPa, the average stress on the $\mathrm{d}$ - $\mathrm{d}$ section is $25.986 \mathrm{MPa}$, and the average stress on the e-e section is $20.499 \mathrm{MPa}$.

\section{Analysis Method of Force Distribution of Four Rows of Bolts}

The installation positions of the four rows of bolts in the fitting joint of outer wing section are shown in Figure 15. Each row of bolts contains two M6 bolts and two M8 bolts. M6 and 


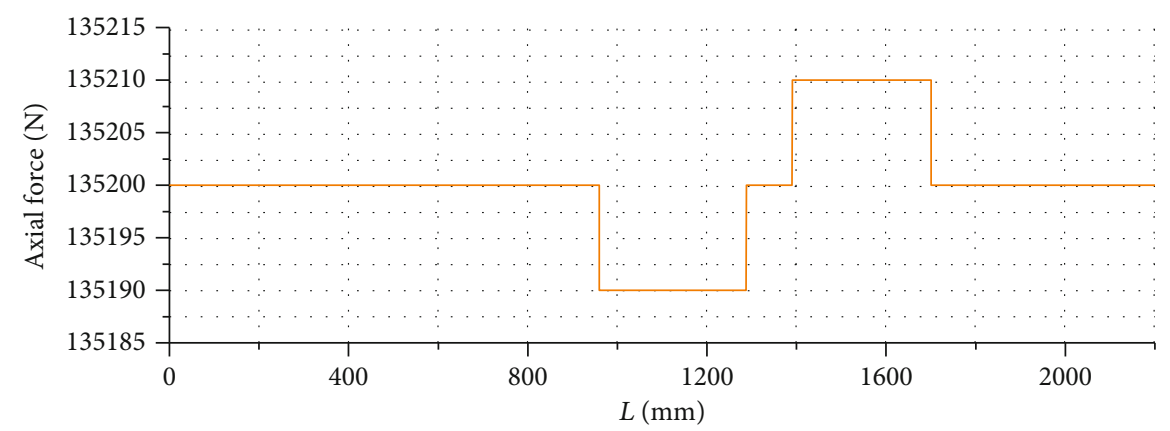

FIGURE 9: Axial force diagram when the gross load is $130 \mathrm{MPa}$.

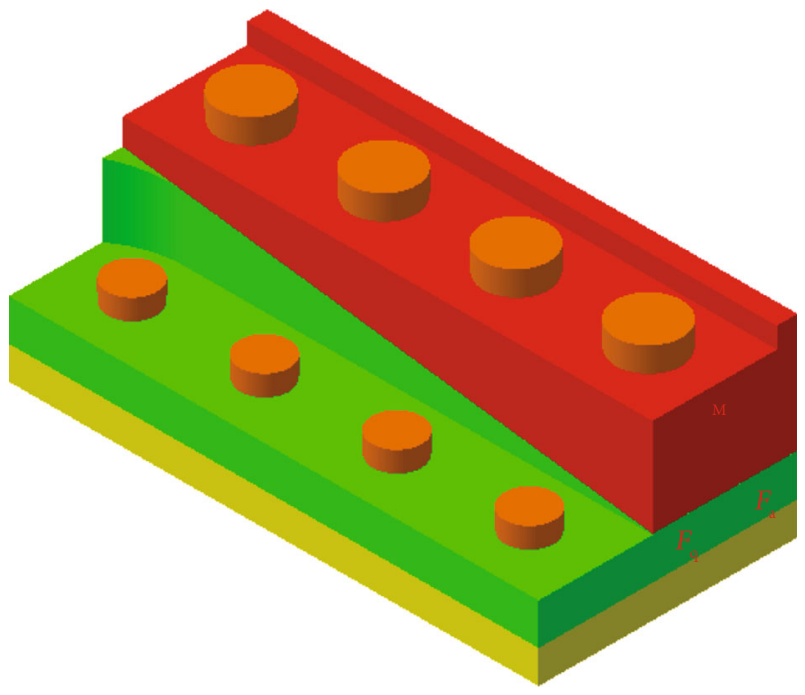

FIgURE 10: The 3D model of the 10th segment model.

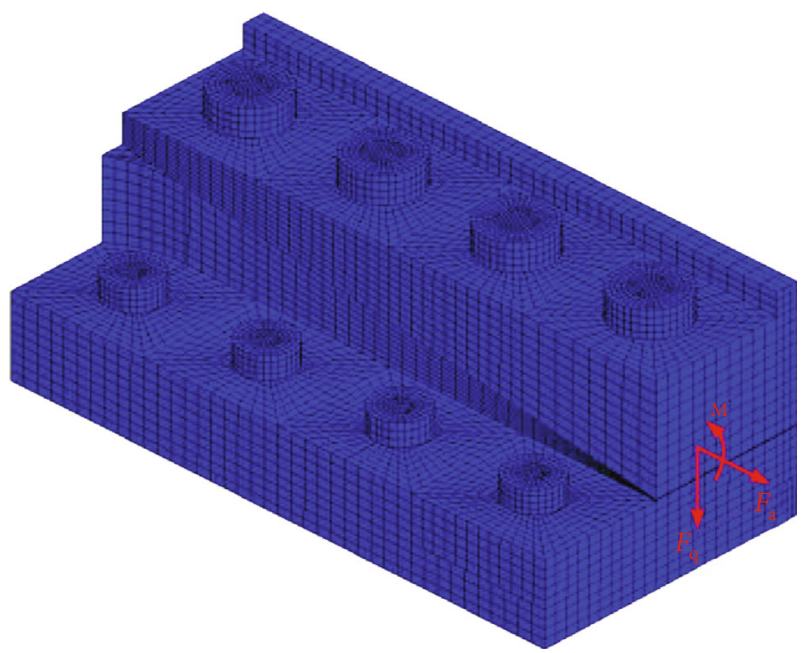

FIgUre 11: The finite element model of the 10th segment model.

M8 bolts are used to install and fix fitting of outer wing section, stringer of outer wing section, and lower panel of outer wing section. It can be seen that the four rows of bolts are very important to the connection and installation of the fitting joint of outer wing section, so it is necessary to analyze the force distribution of the four rows of bolts to ensure the rationality of the structure. Since the fitting joint structure is symmetrical, the force distribution of the four rows of bolts can be obtained by analyzing only one side. The single-sided connection structure of four rows of bolts is shown in Figure 15.

In order to solve this task, a numerical method described in the article [1] is used for reference in this paper. This method obtains the force distribution between the bolt rows based on the average normal stress in the regular section between the bolt rows. The normal stress in the regular section between the bolt rows is derived from the stress-strain state of components in the fitting of outer wing section. The force of a single bolt row is equal to the force difference between the cross-sections on both sides. The advantage of this method is that it takes into account the interaction between the bolt rows and the characteristics between the joint components. It should be pointed out that in the analysis of the model, the averaged stress of the node is set in the Workbench, so it is feasible to use the average stress value of the regular cross-section multiplied by the crosssectional area to obtain the force on the cross-section.

In the connection structure of four rows of bolt, the stringer of outer wing section and the lower panel of outer wing section produce a shear force on bolts under the effect of pulling force and the bolts produce pressure on the fitting of outer wing section after loading. Considering the bolts and the fitting of outer wing section as a whole, the force on the bolts is equivalent to the force on the fitting of outer wing section. In this way, it is only necessary to analyze the effect of the fitting of outer wing section on the bolts, and the force distribution of the bolts can be obtained. Therefore, the force of each row of bolts is calculated as follows:

Force of the first row of bolts: $P_{1}=F_{\mathrm{a}-\mathrm{a}}-F_{\mathrm{b}-\mathrm{b}}$.

Force of the second row of bolts: $P_{2}=F_{\mathrm{b}-\mathrm{b}}-F_{\mathrm{c}-\mathrm{c}}$.

Force of the third row of bolts: $P_{3}=F_{\mathrm{c}-\mathrm{c}}-F_{\mathrm{d}-\mathrm{d}}$.

Force of the fourth row of bolts: $P_{4}=F_{\mathrm{d}-\mathrm{d}}-F_{\mathrm{e}-\mathrm{e}}$.

$F_{\mathrm{a}-\mathrm{a}}$ is the average force on the cross-section $\mathrm{a}-\mathrm{a} ; F_{\mathrm{b}-\mathrm{b}}$ is the average force on the cross-section $b-b ; F_{c-c}$ is the average force on the cross-section $\mathrm{c}-\mathrm{c} ; F_{\mathrm{d}-\mathrm{d}}$ is the average force on the cross-section d-d.

Each bolt row is composed of two M8 bolts and two M6 bolts in the connection structure of four rows of bolts. Due to the different diameters and working positions of the M8 and M6 bolts, the forces distributed on the M8 and M6 bolts are 


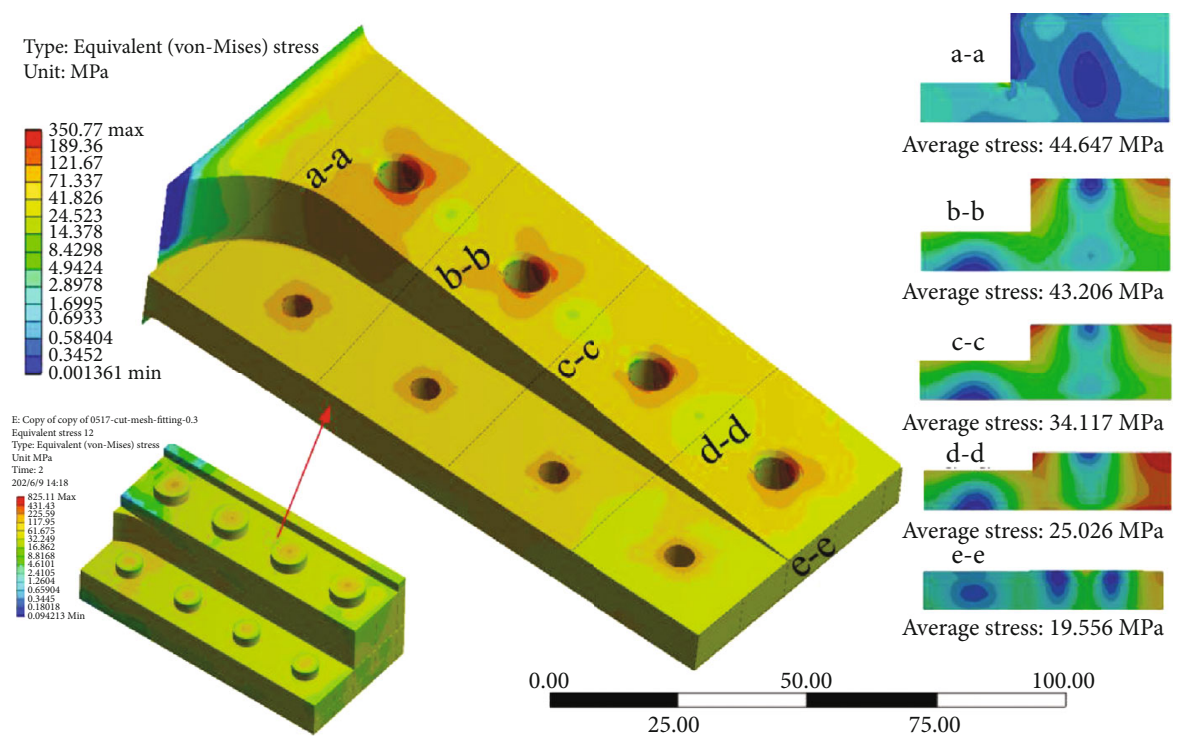

Figure 12: The stress state of the outer wing segment in the 10th segment model.

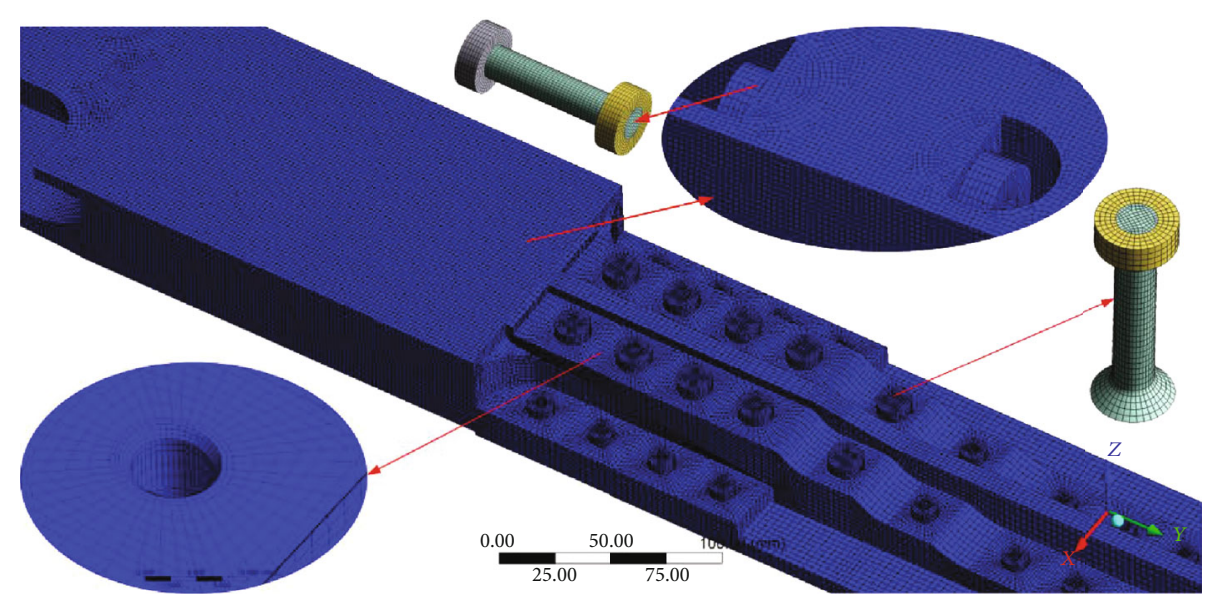

Figure 13: The finite element model of the fitting joint.

different. The force between the bolts is distributed proportionally according to the working area of the bolt which is as follows:

Force of M8 bolts in the $i^{\text {th }}$ row: $P_{8 i}=\left(S_{8 i} /\left(S_{6 i}+S_{8 i}\right)\right) \times P_{i}$. Force of M6 bolts in the $i$ th row: $P_{6 i}=\left(S_{6 i} /\left(S_{6 i}+S_{8 i}\right)\right) \times P_{i}$. $i=1,2,3,4 ; S_{6 i}$ is the working area of M6 bolts in the $i^{\text {th }}$ row; $S_{8 i}$ is the working area of M8 bolts in the $i^{\text {th }}$ row.

\section{Result and Discussion}

The area and average normal stress of each cross-section are shown in Table 3. The area of each cross-section is obtained from the three-dimensional model. The average normal stress 1 is obtained from the result of the step-by-step calculation (Figure 12). The average normal stress 2 is obtained from the result of the entire calculation (Figure 14). The average force on each cross-section can be calculated by formula (1). Therefore, the force distribution between each bolt row is shown in Figure 16. The 1-all curve is the force distribution between the bolt rows obtained by step-by-step calculation, the 2-all curve is the force distribution between the bolt rows obtained by entire calculation, the 1-M8 curve is the force distribution between M8 bolts obtained by step-by-step calculation, the 2-M8 curve is the force distribution between M8 bolts obtained by entire calculation, the 1-M6 curve is the force distribution between M6 bolts obtained by stepby-step calculation, and the 2-M6 curve is the force distribution between M6 bolts obtained by entire calculation.

According to the results of the force of a single row of bolts and M8 or M6 bolts in a four-row bolt connection structure, the force distribution coefficient $R$ between the bolt row and M8 or M6 bolts can be calculated as follows:

Force distribution coefficient between bolt rows: $R_{i}=P_{i} /$ $F_{a}$.

The force distribution coefficient of M8 bolts: $R_{8 i}=P_{8 i} / F_{a}$.

The force distribution coefficient of M6 bolts: $R_{6 i}=P_{6 i} / F_{a}$. $i=1,2,3,4 ; F_{a}$ is the axial force at the end of the 10th segment.

The calculation results of the force distribution coefficient of the bolt rows and the force distribution coefficient 


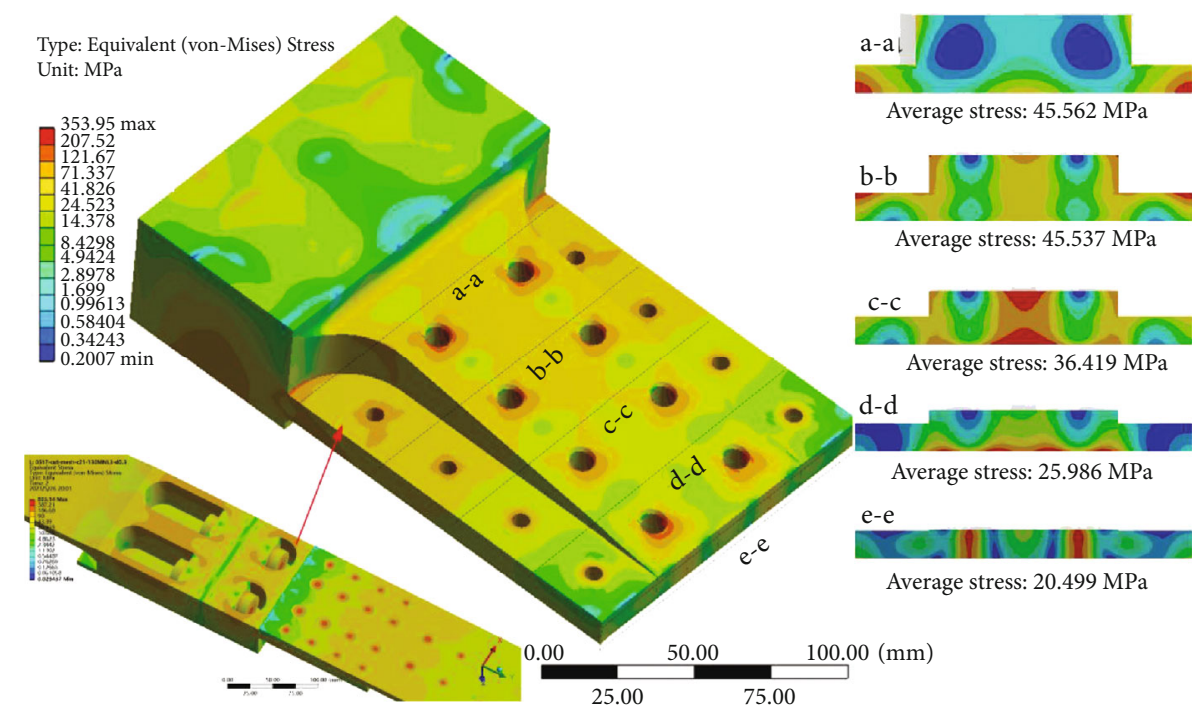

FIGURE 14: The stress state of the fitting of outer wing section.

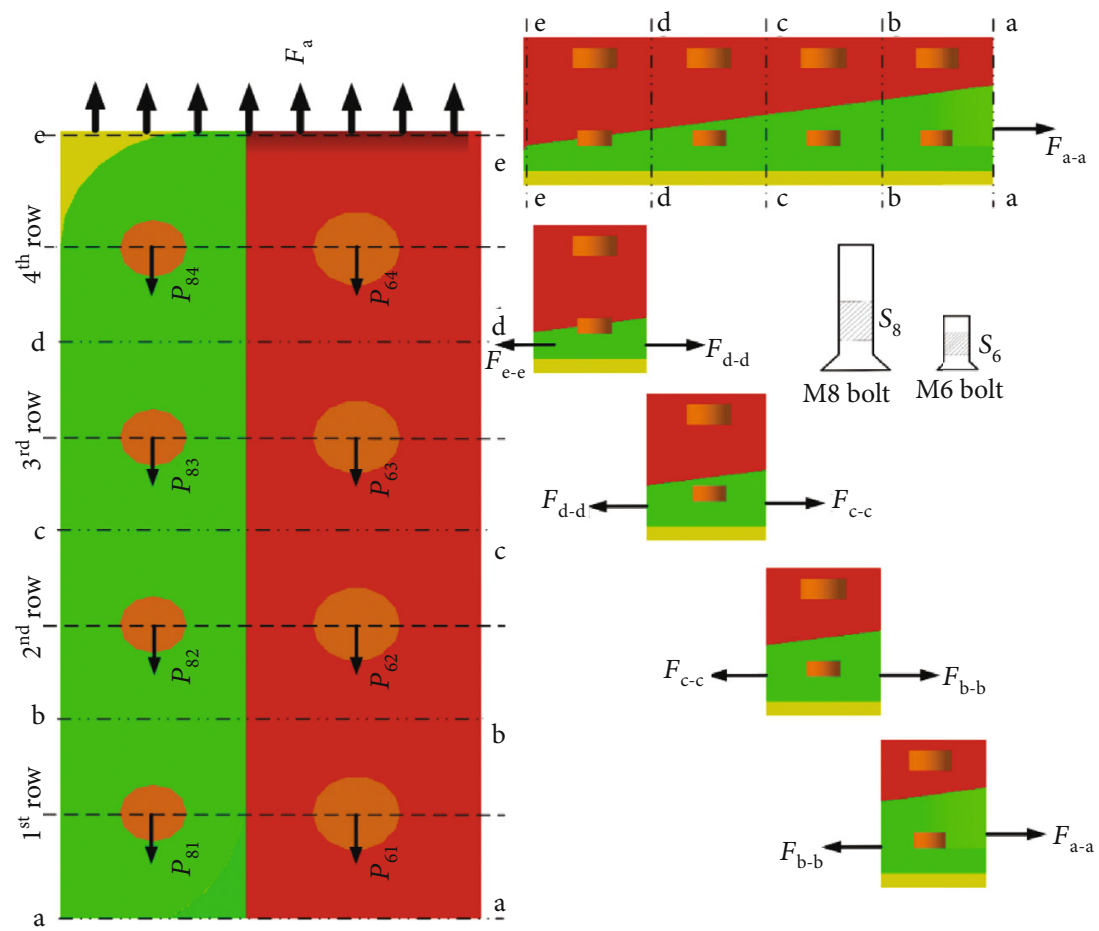

FIgURE 15: The single-sided connection structure of four rows of bolts.

TABLE 3: The area and average normal stress of cross-sections.

\begin{tabular}{lccccc}
\hline Cross-section & a-a & b-b & c-c & d-d & e-e \\
\hline Area $\left(\mathrm{mm}^{2}\right)$ & 2661.43 & 2181 & 1877 & 1573 & 1250 \\
Average normal stress 1 (MPa) & 44.647 & 43.206 & 35.117 & 25.026 & 19.556 \\
Average normal stress 2 (MPa) & 45.562 & 45.531 & 36.419 & 25.986 \\
\hline
\end{tabular}

of a single bolt are shown in Figure 17. The curves of M8 and M6 are the force distribution coefficient between bolt rows, and the force distribution coefficients between the bolt rows are $0.182,0.21,0.197$, and 0.12 . The M8 curve is the force dis- tribution coefficient of M8 bolts, and the force distribution coefficients between the M8 bolts are $0.141,0.155,0.136$, and 0.075 . The M6 curve is the force distribution coefficient of M6 bolts, and the force distribution coefficients between 


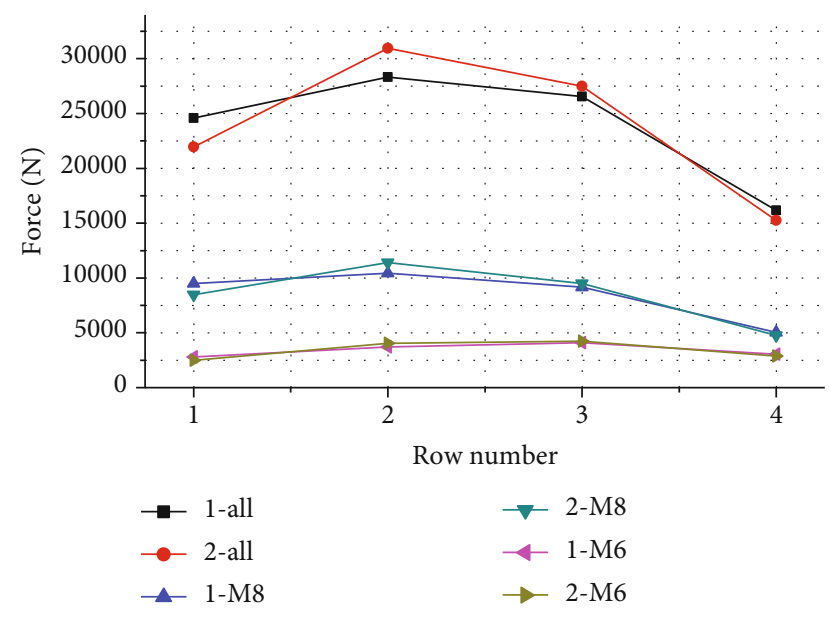

Figure 16: The force distribution between four rows of bolts.

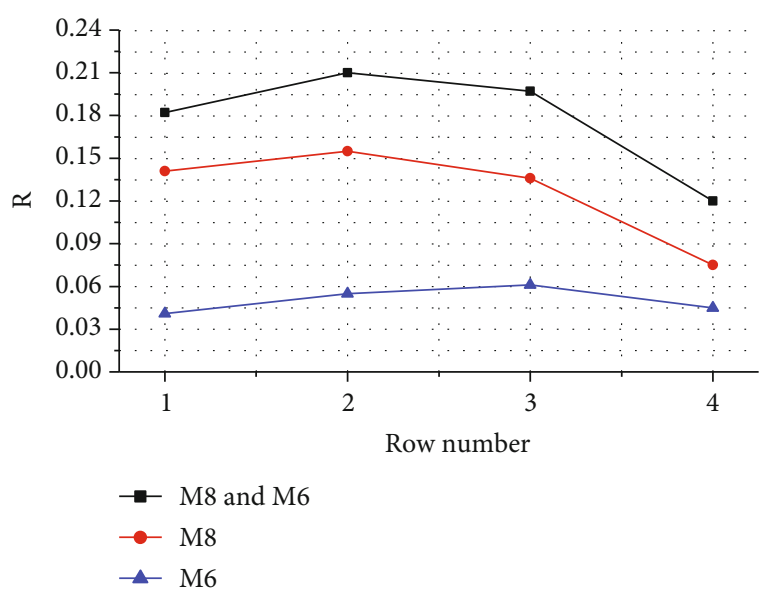

Figure 17: The force distribution coefficient $R$ between four rows of bolts.

the M6 bolts are $0.041,0.055,0.061$, and 0.045 . It can be seen from Figures 16 and 17 that the force between the four rows of bolts is uneven, which is caused by the different areas and positions of the bolts. The sum of the force coefficients of the four rows of bolts is less than 1; this is because part of the force is affected by the structure during transmission, which is used to generate shear and bending moments.

\section{Conclusion}

(1) In a four-row bolted fitting joint, the force distribution coefficients between the bolt rows are 0.182 , $0.21,0.197$, and 0.12 . The force of the four rows of bolts is uneven, which is caused by the different areas and positions of the bolts. The second row of bolts has the most force, and the force distribution coefficient is 0.21 , of which M8 bolts have the most force and the corresponding force distribution coefficient of a single M8 bolt is 0.155 . The fourth row of bolts receives the least force, and the corresponding force distribution coefficient is 0.12 . In the design process of the fitting joint, the structure can be further opti- mized according to the force distribution of the bolts to increase the service life of the fitting joint

(2) A new method for calculating the force distribution between bolts in aircraft fitting joint is developed in this paper. The force distribution between the bolt rows is calculated on the basis of the stress state of the aircraft fitting joint. The step-by-step calculation method is used to calculate the stress-strain state of the fitting joint. Compared with the entire calculation, the step-by-step calculation has the advantages of less calculation amount and faster calculation speed

\section{Data Availability}

The data in the article are from CATIA and Ansys, which are true and effective.

\section{Conflicts of Interest}

The authors declare that there is no conflict of interest regarding the publication of this paper.

\section{Acknowledgments}

This work was supported by the National Scholarship Council of China (No. 201908360296).

\section{References}

[1] E. T. Vasilevsky, A. G. Grebennikov, A. Y. Efremov, and S. P. Svetlichny, "Analysis of the characteristics of the general stress-strain state in the elements of the fitting joint of the wing with the center section," Open Information and Computer Integrated Technologies, vol. 24, pp. 78-89, 2004.

[2] E. T. Vasilevsky, A. G. Grebennikov, A. Y. Efremov, and S. P. Svetlichny, "Methods for determining the characteristics of local stress-strain state in the zone of the monolithic profile of the fitting joint of a wing with a center section," Open Information and Computer Integrated Technologies, vol. 28, pp. 177-193, 2005.

[3] E. T. Vasilevsky, A. G. Grebennikov, A. Y. Efremov, and N. V. Efremova, "The method of integrated design, construction and modeling of a high-resource fitting junction of a wing with a center section of a transport category aircraft," Open Information and Computer Integrated Technologies, vol. 46, pp. 277293, 2010.

[4] W. Xintao, X. Long, and L. Xingke, "Multi-joint and large load wing load processing method," Advances in Aeronautical Science and Engineering, vol. 11, no. 2, pp. 251-255, 2020.

[5] W. Xintao, "Aircraft structural strength test fuselage support design and analysis," Engineering \& Test, vol. 8, no. 5, pp. 104-109, 2019.

[6] Q. Jianbo, N. Yu, X. Wang, and S. Tan, "Failure analysis and improvement of aircraft wing body joint fatigue test piece," Failure Analysis and Prevention, vol. 12, no. 5, pp. 304-309, 2017.

[7] Z. Yanqin and W. Shili, "Research on the connection of composite wing roots," Mechanical Engineer, vol. 5, pp. 99-102, 2017. 
[8] X. Wang, Z. Bin, L. Yonggang, and Y. Zhufeng, "Non-linear finite element analysis of wing and fuselage butt joint," Computer Simulation, vol. 26, no. 7, pp. 37-40, 2009.

[9] L. Liu and K. Chen, "Global-local finite element stress analysis of thick laminate multi-bolt joints in large-scale structures," Finite Elements in Analysis and Design., vol. 75, pp. 31-37, 2013.

[10] B. Egan, C. T. McCarthy, M. A. McCarthy, and R. M. Frizzell, "Stress analysis of single-bolt, single-lap, countersunk composite joints with variable bolt-hole clearance," Composite Structures, vol. 94, no. 3, pp. 1038-1051, 2012.

[11] F. Ascione, "A preliminary numerical and experimental investigation on the shear stress distribution on multi-row bolted FRP joints," Mechanics Research Communications., vol. 37, no. 2, pp. 164-168, 2010.

[12] J. Xiang, S. Zhao, D. Li, and Y. Wu, "An improved spring method for calculating the load distribution in multi-bolt composite joints," Composites Part B: Engineering., vol. 117, pp. 18, 2017.

[13] N. Konkong and K. Phuvoravan, “An analytical method for determining the load distribution of single-column multibolt connection," Advances in Civil Engineering, vol. 2017, Article ID 1912724, 19 pages, 2017.

[14] V. A. Boguslayev and A. G. Grebenikov, Concept of Development of Up-to-Date Jet Regional Passenger Aircraft, ANTONOV Company, 2020. 